Physics

Physics Research Publications

\title{
Magnetic measurements on ferromagnetic behavior in the bulk II-VI diluted magnetic semiconductor Zn1-xCrxTe
}

T. M. Pekarek D. J. Arenas $\quad$ B. C. Crooker
I. Miotkowski
A. K. Ramdas

This paper is posted at Purdue e-Pubs.

http://docs.lib.purdue.edu/physics_articles/413 


\title{
Magnetic measurements on ferromagnetic behavior in the bulk II-VI diluted magnetic semiconductor $\mathrm{Zn}_{1-x} \mathrm{Cr}_{x} \mathrm{Te}$
}

\author{
T. M. Pekarek ${ }^{\mathrm{a})}$ and D. J. Arenas \\ Department of Chemistry and Physics, University of North Florida, Jacksonville, Florida 32224 \\ B. C. Crooker \\ Department of Physics, Fordham University, Bronx, New York 10458
}

I. Miotkowski and A. K. Ramdas

Department of Physics, Purdue University, West Lafayette, Indiana 47907

(Presented on 8 January 2004)

\begin{abstract}
Magnetic measurements on the ferromagnetic behavior in the bulk II-VI diluted magnetic semiconductor $\mathrm{Zn}_{1-x} \mathrm{Cr}_{x} \mathrm{Te}$ were made on two $x=0.0033$ single crystals taken from different regions of the same boule. Ferromagnetism was verified in both samples by an Arrott plot analysis with a transition temperature at $365 \mathrm{~K}$ (well above room temperature). For both samples at room temperature, the coercive field is $\sim 0.0100 \mathrm{~T}$ and the remanent magnetization is $23 \%$ of the saturated value. The similarity in the observed ferromagnetic behavior between the two samples suggests that a stable $\mathrm{Cr}_{y} \mathrm{Te}_{z}$ or possibly $\mathrm{Zn}_{x} \mathrm{Cr}_{y} \mathrm{Te}_{z}$ precipitate phase is responsible, although a Cr-rich region in the bulk $\mathrm{Zn}_{1-x} \mathrm{Cr}_{x} \mathrm{Te}$ itself cannot currently be conclusively ruled out as the source. (C) 2004 American Institute of Physics. [DOI: 10.1063/1.1689431]
\end{abstract}

\section{INTRODUCTION}

The II-VI diluted magnetic semiconductors (DMS) have been the topic of many studies over the past two decades. ${ }^{1}$ Many of these studies were driven by an interest in combining semiconductors with magnetic materials. The field of spintronics, ${ }^{2}$ in particular, would be significantly advanced by the discovery of new ferromagnetic semiconducting materials with transitions significantly above room temperature.

In previous work, we reported on a minority ferromagnetic phase in a bulk $\mathrm{Zn}_{1-x} \mathrm{Cr}_{x} \mathrm{Te}$ crystal grown by the vertical Bridgman method. ${ }^{3}$ We found a transition temperature slightly above $300 \mathrm{~K}$ for the ferromagnetic phase. At the time, we ascribed the ferromagnetic signal to a pure $\mathrm{CrTe}$ minority phase. For the purpose of this previous work, the observed ferromagnetic signal was a background feature that was characterized sufficiently to allow for its subtraction.

However, recent work by Saito et al. on molecular beam epitaxy (MBE) grown epitaxial films of $\mathrm{Zn}_{1-x} \mathrm{Cr}_{x} \mathrm{Te}$ found that an $x=0.20$ sample was ferromagnetic ${ }^{4,5}$ with a similar transition temperature and magnetic properties. This result, combined with other work by Saito et al. on the ferromagnetic properties of an $x=0.035 \mathrm{Zn}_{1-x} \mathrm{Cr}_{x} \mathrm{Te}$ epitaxial film with a $T_{c}$ around $15 \mathrm{~K},{ }^{6,7}$ raised the possibility that our minority phase was instead a region of enhanced $\mathrm{Cr}$ concentration within our bulk $\mathrm{Zn}_{1-x} \mathrm{Cr}_{x} \mathrm{Te}$ crystal.

In this paper we report on magnetization measurements on two additional samples removed from different regions of the original boule. An Arrott plot analysis ${ }^{8}$ was conducted to confirm the ferromagnetic behavior and determine the Curie temperature. Additional features of the ferromagnetic behav-

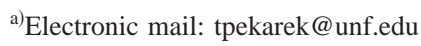

ior were characterized for comparisons between different bulk crystals and the reported epitaxial films.

\section{EXPERIMENTAL DETAILS}

Single-crystalline $\mathrm{Zn}_{1-x} \mathrm{Cr}_{x} \mathrm{Te}$ samples were grown by the vertical Bridgman method. Three samples (A, B, and C) were taken from the same boule, with a nominal concentration of $x=0.005$. Atomic absorption spectroscopy (AAS) was performed on sample A yielding a value of $x=0.0035$ $\pm 0.0005{ }^{3}$ With this concentration for sample $\mathrm{A}$ as a reference, the concentration of samples $\mathrm{B}$ and $\mathrm{C}$ is determined using the ratio of the magnetization at $5 \mathrm{~K}$ and $6 \mathrm{~T}$. This yields values for the concentration for samples $\mathrm{B}$ and $\mathrm{C}$ of $x=0.0033$ and $x=0.0033$, respectively.

Magnetization measurements on sample A were reported previously at temperatures between 2 and $300 \mathrm{~K}$ and in fields up to $6 \mathrm{~T}^{3}$ Samples B and C were measured in a new Quantum Design MPMS XL7 superconducting quantum interference device (SQUID) magnetometer at temperatures between 2 and $400 \mathrm{~K}$ and in fields up to $7 \mathrm{~T}$. The diamagnetic signal due to the host $\mathrm{ZnTe}$ has been subtracted.

It is known that $\mathrm{Zn}_{1-x} \mathrm{Cr}_{x} \mathrm{Te}$ crystals preferentially cleave along the (110) direction. Samples A, B, and C were all mounted along this cleaved direction. The (110) direction was verified by the standard Laue diffraction technique for samples $\mathrm{A}$ and $\mathrm{C}$.

\section{EXPERIMENTAL RESULTS AND DISCUSSION}

Hysteresis loops in the magnetization for samples B and C were taken at a series of temperatures from 2 to $400 \mathrm{~K}$ with a maximum field of $\pm 7 \mathrm{~T}$. Representative hysteresis loops for sample B are shown in Fig. 1(a) at 5, 300, and 400 

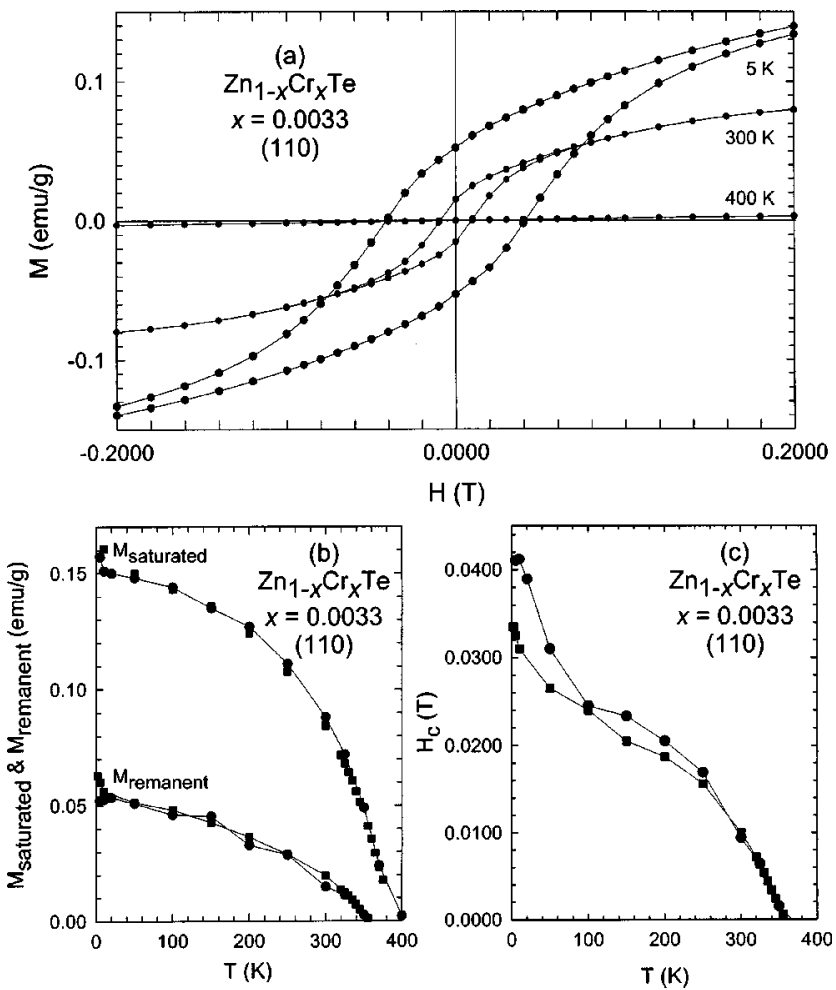

FIG. 1. (a) Magnetization vs field measurements for $\mathrm{Zn}_{1-x} \mathrm{Cr}_{x} \mathrm{Te}(x$ $=0.0033$ ) at 5,300 , and $400 \mathrm{~K}$ are shown as circles for sample B. The lines are a guide to the eye. Hysteresis is clearly visible at 5 and $300 \mathrm{~K}$ but is not at $400 \mathrm{~K}$. (b) The saturated and remanent magnetization vs temperature show the same functional behavior for both samples. The data for sample $\mathrm{C}$ have been scaled in Fig. 1(b) by the ratio of the $0 \mathrm{~K}$ saturated ferromagnetic signals, a factor of 2.62 , to facilitate a comparison with sample B. (c) The coercive field is plotted vs temperature for samples B and C. Samples B and $\mathrm{C}$ are shown as circles and squares, respectively, in (b) and (c).

$\mathrm{K}$. Hysteresis is clearly observed at $300 \mathrm{~K}$ and persists up to $350 \mathrm{~K}$. Above $\sim 355 \mathrm{~K}$, any hysteresis cannot be resolved within the uncertainties of the instrument.

Linear fits to the descending branch of the hysteresis loops between 0.8 and $1.5 \mathrm{~T}$ were used to determine the saturated ferromagnetic signal of each sample. Extrapolating to $0 \mathrm{~K}$, the saturated ferromagnetic signal is $0.055,0.152$, and $0.058 \mathrm{emu} / \mathrm{g}$ for samples $\mathrm{A}, \mathrm{B}$, and $\mathrm{C}$, respectively. The saturated ferromagnetic signal as a function of temperature for samples B and C is shown in Fig. 1(b) [note the data for sample $C$ in Fig. 1(b) have been scaled by the ratio of the 0 $\mathrm{K}$ saturated ferromagnetic signals, a factor of 2.62, to facilitate a comparison with sample B]. The remanent magnetization for both samples is also shown in Fig. 1(b). After scaling the data for sample $\mathrm{C}$, both samples are seen to exhibit the same temperature dependence for the remanent and saturated ferromagnetic signal.

The coercive field $H_{c}$ versus temperature for samples B and $\mathrm{C}$ is shown in Fig. 1(c). $H_{c}$ is large at low temperatures and persists up to $T_{c}$. The coercive field as a function of temperature follows the same general behavior from $T_{c}$ down to $100 \mathrm{~K}$. Below $100 \mathrm{~K}, H_{c}$ varies by up to $33 \%$ at 10 $\mathrm{K}$ where $H_{c}$ reaches $0.0412 \mathrm{~T}$ for sample $\mathrm{B}$.

To confirm that the hysteresis is due to ferromagnetism and to determine the transition temperature $T_{c}$, we analyzed magnetization data from 1 to $7 \mathrm{~T}$ following the work of

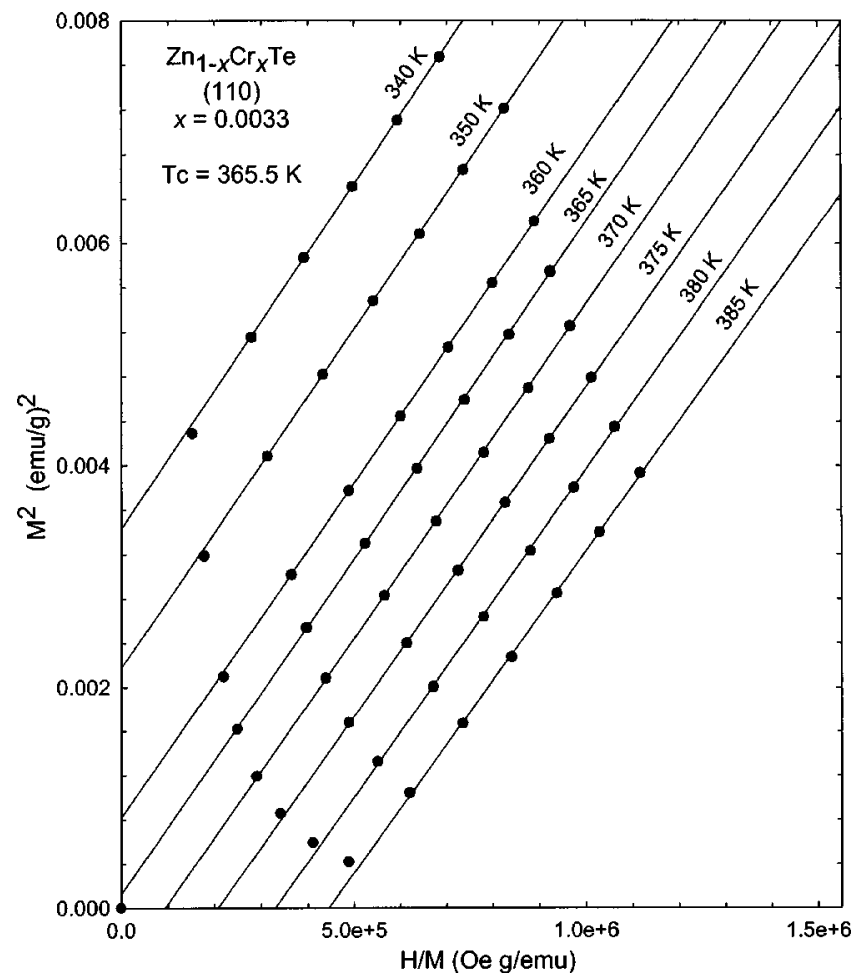

FIG. 2. Magnetization data for sample B taken at 340-385 K in fields between 1 and $7 \mathrm{~T}$ are shown as circles and plotted as $M^{2}$ vs $H / M$. At $T_{c}$ for a ferromagnetic transition, the intercept $1 / \chi_{0}$ on the $H / M$ axis passes through the origin. The lines are linear fits to the high field data at a set temperature. For this sample $T_{c}$ is $365.5 \mathrm{~K}$.

Arrott. $^{8}$ For temperatures around $365 \mathrm{~K}$, we observed straight lines in a plot of $M^{2}$ vs $H / M$. Data for sample B are shown in Fig. 2. As we approach $365 \mathrm{~K}$ from above and below, the intercept $1 / \chi_{0}$ on the $H / M$ axis approaches the origin. Slight curvature toward the origin can be observed in data taken below $\sim 1 \mathrm{~T}$ at 340 and $385 \mathrm{~K}$ as expected. Extrapolating $1 / \chi_{0}$ vs $T$ (Fig. 3), the value of $T_{c}=365.5 \mathrm{~K}$ is

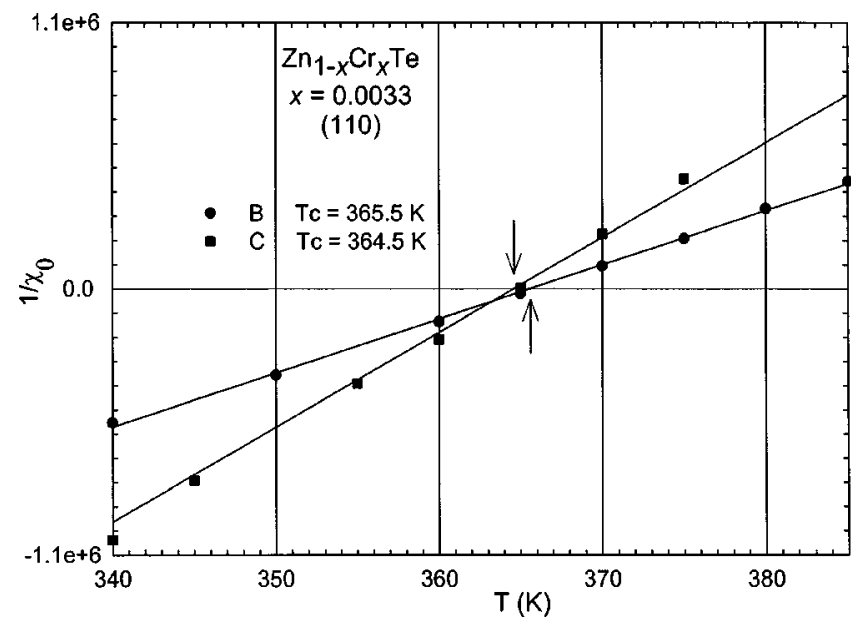

FIG. 3. Intercept $1 / \chi_{0}$ for each set temperature in Fig. 2 is plotted as circles vs set temperature. The temperature where $1 / \chi_{0}$ goes to zero (the $x$ intercept) corresponds to $T_{c}$. For sample $\mathrm{B}$, shown by the circles, $T_{c}$ $=365.5 \mathrm{~K}$. The corresponding data for sample $\mathrm{C}$ are plotted as squares and yield $T_{c}=364.5 \mathrm{~K}$. 
obtained from the point at which $1 / \chi_{0}$ goes to zero. Similar data were observed for sample $\mathrm{C}$ (plotted as squares in Fig. 3) giving $T_{c}=364.5 \mathrm{~K}$.

The magnetization in $0.6 \mathrm{~T}$ above $T_{c}$ was also plotted as $H / M$ vs $T$ (not shown). From a Curie-Weiss fit to the data between 380 and $400 \mathrm{~K}$, we obtain a temperature intercept of 365.1 and $364.7 \mathrm{~K}$ for samples B and C, respectively. These values are in excellent agreement to the values of $T_{c}$ determined from the Arrott plot analysis.

Having established that ferromagnetism exists in these bulk $\mathrm{Zn}_{1-x} \mathrm{Cr}_{x} \mathrm{Te}$ crystals with a transition temperature at $365 \mathrm{~K}$ (well above room temperature), the obvious remaining issue is to determine exactly what within the crystal is responsible for the ferromagnetic signal. Is the ferromagnetism due to regions of enhanced $\mathrm{Cr}$ concentration in the bulk II-VI DMS $\mathrm{Zn}_{1-x} \mathrm{Cr}_{x} \mathrm{Te}$ itself or is a precipitate within the host II-VI DMS responsible? Either way, we have a potentially useful system. If regions within the bulk II-VI DMS $\mathrm{Zn}_{1-x} \mathrm{Cr}_{x} \mathrm{Te}$ itself are ferromagnetic, then the higher $T_{c}$ implies local $\mathrm{Cr}$ concentrations exceeding the value of $x$ $=0.20$ obtained by Saito et al. for the epitaxial film. Instead, if the observed ferromagnetism is due to precipitates of $\mathrm{Cr}_{y} \mathrm{Te}_{z}{ }^{9}$ or possibly $\mathrm{Zn}_{x} \mathrm{Cr}_{y} \mathrm{Te}_{z}$ then we have shown it is possible to obtain ferromagnetic precipitates in a II-VI DMS host that was shown by Saito et al. ${ }^{4}$ to also exhibit ferromagnetism. In this case, one can envision possible spintronic applications based on the interplay between carriers, ferromagnetism in the DMS, and ferromagnetic precipitates.

In comparing the two samples, it is worth emphasizing that the fraction of $\mathrm{Cr}$ atoms contributing to the ferromagnetic and singlet magnetization features is significantly different for samples B and C. Sample B has $42 \%$ of the Cr contributing to the ferromagnetic signal while sample $\mathrm{C}$ has only $16 \%$. And yet, the ferromagnetic behavior exhibited by the two samples is very similar. This suggests that the observed behavior arises from a stable precipitate phase that is compatible with the host $\mathrm{Zn}_{1-x} \mathrm{Cr}_{x} \mathrm{Te}$ lattice. It is known that such ferromagnetic precipitates can be formed in a host semiconductor. For example, ferromagnetic $\mathrm{Fe}_{3} \mathrm{GaAs}$ clusters have been observed in GaAs where $\mathrm{Fe}_{3} \mathrm{GaAs}$ grains form well-defined boundaries canted with respect to the GaAs host. ${ }^{10-12}$ Such a stable stoichiometry within the semiconductor host could form in regions throughout the boule and exhibit the same $T_{c}$ and temperature dependence for the saturated and remanent magnetization. Because of different cluster sizes, one might expect the temperature dependence on $H_{c}$ to vary somewhat throughout the boule.

Ferromagnetism arising from regions of enhanced $\mathrm{Cr}$ concentration within the bulk $\mathrm{Zn}_{1-x} \mathrm{Cr}_{x} \mathrm{Te}$ is unlikely to explain the sharp $T_{c}$ and similar magnetic properties we ob- serve in these samples. One would expect that concentration gradients throughout the boule would produce different values of $T_{c}$ in different regions. In the present case, it is difficult to envision finding two samples taken from different locations within a boule with a nominal concentration of $x$ $=0.005$ containing the same local concentration in excess of $x=0.20$ that would be required to produce ferromagnetic regions with values for $T_{c}$ that are so close to the same value.

\section{CONCLUSIONS}

Ferromagnetism was verified in two samples of the bulk II-VI diluted magnetic semiconductor $\mathrm{Zn}_{1-x} \mathrm{Cr}_{x} \mathrm{Te}(x$ $=0.0033$ ) by an Arrott plot analysis with a transition temperature at $365 \mathrm{~K}$ (well above room temperature). For both samples at room temperature, the coercive field is $\sim 0.0100 \mathrm{~T}$ and the remanent magnetization is $23 \%$ of the saturated value. The observed ferromagnetic behavior is believed to arise from precipitates of $\mathrm{Zn}_{x} \mathrm{Cr}_{y} \mathrm{Te}_{z}$ within the II-VI DMS host, although the bulk $\mathrm{Zn}_{1-x} \mathrm{Cr}_{x} \mathrm{Te}$ itself cannot currently be conclusively ruled out as the source.

\section{ACKNOWLEDGMENTS}

This research was supported by the Donors of the American Chemical Society Petroleum Research Fund PRF No. 40209-B5M, a Purdue University Academic Reimbursement Grant, and by the National Science Foundation (NSF) Grant Nos. DMR-03-05653, DMR-01-02699, and ECS-0129853.

${ }^{1}$ See, for example, Semiconductors and Semimetals, edited by J. K. Furdyna and J. Kossut (Academic, Boston, 1988), Vol. 25.

${ }^{2}$ S. A. Wolf, D. D. Awschalom, R. A. Buhrman, J. M. Daughton, S. von Molnar, M. L. Roukes, A. Y. Chtchelkanova, and D. M. Treger, Science 294, 1488 (2001).

${ }^{3}$ T. M. Pekarek, J. E. Luning, I. Miotkowski, and B. C. Crooker, Phys. Rev. B 50, 16914 (1994).

${ }^{4}$ H. Saito, V. Zayets, S. Yamagata, and K. Ando, Phys. Rev. Lett. 90, 207202 (2003).

${ }^{5}$ H. Saito, V. Zayets, S. Yamagata, and K. Ando, J. Appl. Phys. 93, 6796 (2003)

${ }^{6}$ H. Saito, W. Zaets, S. Yamagata, Y. Suzuki, and K. Ando, J. Appl. Phys. 91, 8085 (2002).

${ }^{7}$ H. Saito, V. Zayets, S. Yamagata, and K. Ando, Phys. Rev. B 66, 081201(R) (2002).

${ }^{8}$ A. Arrott, Phys. Rev. 108, 1394 (1957).

${ }^{9}$ Solid State Physics Literature Guides, edited by T. F. Connolly (IFI/ Plenum, New York, 1972), Vol. 5.

${ }^{10}$ J. C. P. Chang, N. Otsuka, E. S. Harmon, M. R. Melloch, and J. M. Woodall, Appl. Phys. Lett. 65, 2801 (1994).

${ }^{11}$ T. M. Pekarek, B. C. Crooker, D. D. Nolte, J. Deak, M. McElfresh, J. C. P. Chang, E. S. Harmon, M. R. Melloch, and J. M. Woodall, J. Magn. Magn. Mater. 169, 261 (1997).

${ }^{12}$ D. T. McInturff, E. S. Harmon, J. C. P. Chang, T. M. Pekarek, and J. M. Woodall, Appl. Phys. Lett. 69, 1885 (1996). 